\title{
SHEAF COHOMOLOGY OF LOCALLY COMPACT TOTALLY DISCONNECTED SPACES
}

\author{
ROGER WIEGAND 1
}

Pierce [4] has developed a sheaf-theoretic representation of rings and modules, which proves to be particularly useful in the study of commutative regular rings. In the present paper we apply this representation theory to the study of locally compact, totally disconnected spaces. The central result is Theorem 2.1, which represents the sheaf cohomology in terms of Ext's. In $\$ 4$ we show that sheaf cohomology and Cech cohomology coincide for the spaces under consideration, and in $\S 5$ we show that there exists a wide class of totally disconnected spaces with interesting cohomology.

We make the standing assumption that all rings have identity and that all $R$-modules are unitary left $R$-modules.

1. Preliminaries. In this section we outline briefly and without proofs the representation theory which is developed in [4, Part I]. All notation and terminology established here will be preserved throughout the paper.

If $R$ is an arbitrary ring, let $B(R)$ denote the set of central idempotents of $R$, with the natural Boolean algebra structure:

$$
e_{1} \vee e_{2}=e_{1}+e_{2}-e_{1} e_{2}, \quad e_{1} \wedge e_{2}=e_{1} e_{2} .
$$

Let $X(R)$ denote the maximal ideal space of $B(R)$. Then $X(R)$ is a Boolean space, i.e., a compact, totally disconnected Hausdorff space. For each $e \in B(R)$, let $N(e)$ be the corresponding open-and-closed subset of $X(R)$. ( $N(e)$ is the set of maximal ideals not containing $e$.) If $I$ is any ideal of the Boolean algebra $B(R)$, let $I^{-}=\{r e \mid r \in R, e \in I\}$. Then $I^{-}$is an ideal of the ring $R$.

Let $A$ be any $R$-module. For each $M \in X(R)$, let $a_{M}$ be the abelian group $A / M-A$. Let $Q(A)$ be the disjoint union $\cup\left\{\mathfrak{Q}_{M}: M \in X(R)\right\}$. For each $a \in A$, define a function $\tau_{a}: X(R) \rightarrow Q(A)$ by letting $\tau_{a}(M)$ be the coset of $a$ in $A / M-A$. The sets $\tau_{a}(N(e)), a \in A, e \in B(R)$, form a basis for a topology on $Q(A)$, and with this topology $a(A)$ is a sheaf of abelian groups over $X(R)$. In the special case $A=R$, we write $\Re(R)$ instead of $Q(R)$, and $\sigma_{r}$ instead of $\tau_{r}$. Then $R(R)$ is a sheaf of

Received by the editors September 26, 1967.

1 The material in this paper is part of a dissertation submitted to the University of Washington in partial fulfillment of the requirements for the degree of Doctor of Philosophy. The research was supported in part by an NSF Graduate Fellowship. 
rings, and the natural $R / M^{-}$-module structure on $A / M^{-} A$ induces an $Q(R)$-Module structure on $Q(A)$.

Theorem 1.1 (PIERCE). Let $R$ be a ring and let $A$ be an $R$-module. (i) The mapping $r \rightarrow \sigma_{r}$ is a ring isomorphism between $R$ and $\Gamma(X(R), R(R))$. (ii) The mapping $a \rightarrow \tau_{a}$ is an abelian group isomorphism between $A$ and $\Gamma(X(R), a(A))$. (iii) $\tau_{r a}=\sigma_{r} \tau_{a}$, for each $r \in R, a \in A$. (iv) If $\phi: A \rightarrow B$ is an R-homomorphism, there exists a unique $R(R)$-homomorphism $\phi_{0}: Q(A) \rightarrow Q(B)$, such that $\phi_{0} \circ \tau_{a}=\tau_{\phi(a)}$, for each $a \in A$. (v) The correspondences $A \rightarrow Q(A), \phi \rightarrow \phi_{0}$ define an equivalence between $R$-mod, the category of $R$-modules, and $R(R)$-Mod, the category of $R(R)$-Modules.

2. The isomorphism theorem. An ideal of a ring $R$ is regular if it is generated by central idempotents. For each regular ideal $J$, let $U[J]=U\{N(e) \mid e \in J \cap B(R)\}$. It is straightforward to check [4] that the mapping $J \rightarrow U[J]$ is an isomorphism from the lattice of regular ideals of $R$ to the lattice of open subsets of $X(R)$.

THEOREM 2.1. Let $A$ be an $R$-module and let $J$ be a regular ideal of $R$. Then, for each $n \geqq 0, \operatorname{Ext}_{R}^{n}(J, A)$ and $H^{n}(U[J] ; a(A))$ are naturally isomorphic. ${ }^{2}$

The proof will be given in the next section.

Lemma 2.2. Let $e \in B(R), a \in A$. Then $e a=0$ if and only if $\tau_{a} \mid N(e)$ $=0$.

Proof. Observe that $\sigma_{e} \mid N(e)=1$, and $\sigma_{e} \mid(X(R)-N(e))=0$. Hence $\sigma_{e} \tau_{a}=0$ if and only if $\tau_{a} \mid N(e)=0$. The desired result now follows from Theorem 1.1, (ii), (iii).

Lemma 2.3. For each $f \in \operatorname{Hom}_{R}(J, A)$, there exists a unique section $\theta(f) \in \Gamma(U[J], Q(A))$ such that

$$
\theta(f)\left|N(e)=\tau_{f(e)}\right| N(e)
$$

for each $e \in J \cap B(R)$.

Proof. $\theta(f)$ is defined locally by $(2.1)$. Since the sets $N(e)$ form an open cover of $U[J]$, we need only check that the sections $\tau_{f\left(e_{1}\right)}$ and $\tau_{f\left(e_{2}\right)}$ agree on $N\left(e_{1}\right) \cap N\left(e_{2}\right)$, whenever $e_{1}$ and $e_{2}$ are in $J \cap B(R)$. Now $e_{1} e_{2}\left(f\left(e_{1}\right)-f\left(e_{2}\right)\right)=0$; hence $\tau_{f\left(e_{1}\right)-f\left(e_{2}\right)} \mid N\left(e_{1} e_{2}\right)=0$, by Lemma 2.2. But $N\left(e_{1} e_{2}\right)=N\left(e_{1}\right) \cap N\left(e_{2}\right)$, and the required identity now follows from Theorem 1.1 (ii).

2 To be more precise we should write $H^{n}(U[J] ; Q(A) \mid U[J])$, where $Q(A) \mid U[J]$ is regarded only as a sheaf of abelian groups. 
Lemma 2.4. The mapping $\theta: \operatorname{Hom}_{R}(J, A) \rightarrow \Gamma(U[J], a(A))$ is an abelian group isomorphism.

Proof. Using (2.1) and Theorem 1.1 (ii), we see that $\theta$ is a homomorphism. Moreover, Lemma 2.2 implies that $\theta$ is one-to-one. To show that $\theta$ is onto, let $s \in \Gamma(U[J], Q(A))$ be arbitrary. For each $e \in J \cap B(R)$, let $f(e)$ denote the unique element of $A$ such that $\tau_{f(e)}=\sigma_{e} s$. Using Lemma 2.2 and Theorem 1.1, one can easily verify the relations

$$
e f(e)=f(e), \quad e_{1} f\left(e_{2}\right)=e_{2} f\left(e_{1}\right)
$$

for $e, e_{1}, e_{2} \in J \cap B(R)$. Using (2.2) several times, one checks that if $r_{1} e_{1}=r_{2} e_{2},\left(r_{i} \in R\right)$, then $r_{1} f\left(e_{1}\right)=r_{2} f\left(e_{2}\right)$. It follows that the function $f: J \cap B(R) \rightarrow A$ can be extended to a unique $R$-homomorphism $\bar{f}: J \rightarrow A$. Clearly $\theta(\bar{f})=s$, and the proof is complete.

3. Proof of Theorem 2.1. Throughout this section, $J$ is a fixed regular ideal of a ring $R$. For each $R$-module $A$, let $\theta_{A}: \operatorname{Hom}_{R}(J, A)$, $\rightarrow \Gamma(U[J], a(A))$ be the isomorphism constructed in Lemma 2.3. Let $A$ and $B$ be $R$-modules, and let $\phi \in \operatorname{Hom}_{R}(A, B)$. It is easily checked, using (2.1) and Theorem 1.1 (iv), that the following diagram commutes:

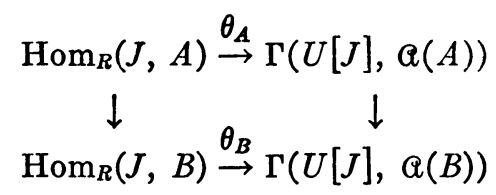

where the vertical arrows are the homomorphisms induced by $\phi$ and $\phi_{0}$, respectively.

We now regard $\operatorname{Hom}_{R}(J, \quad)$ and $\Gamma(U[J], Q())$ as covariant functors from $R$-mod to $G$, the category of abelian groups. Lemma 2.4 and the above diagram provide a natural equivalence $\theta$ between these functors. The rest of the proof is essentially a formality. The simplest approach is to use the following three properties of the sheaf cohomology of a space $X$, which are verified in [2, Proposition 3.2.1]:

(i) The system of functors $H^{n}(X ;)_{n \geq 0}$ forms an exact $\partial$-functor from $g^{X}$, the category of sheaves of abelian groups over $X$, to $G$.

(ii) $H^{0}(X ;)=\Gamma(X$,$) .$

(iii) $H^{p}(X ;)$ is effaceable, for each $p>0$.

Now the functors $A \rightarrow Q(A)$, from $R$-mod to $R(R)$-Mod, and $Q(A)$ $\rightarrow \mathfrak{Q}(A) \mid U[J]$, from $R(R)$-Mod to $g^{U[J]}$, are both exact. Consequently, the system of functors $H^{n}(U[J] ; Q())_{n \succeq 0}$ forms an exact $\partial$-functor from $R$-mod to $g$. Now let $p>0$. We shall show that the 
functor $H^{p}(U[J] ; a())$ is effaceable. Let $A$ be an arbitrary $R$-module, and let $Q$ be an injective module containing $A$. Then $Q(Q)$ is injective (by Theorem $1.1(\mathrm{v})$ ), and hence flabby. But then $Q(Q) \mid U[J]$ is flabby, so that $H^{p}(U[J] ; Q(Q))=0$. It now follows from [2, Proposition 2.2.1] that the sequence $H^{n}(U[J] ; Q())_{n \geq 0}$ is a universal $\partial$-functor from $R$-mod to $\mathrm{g}$. But $\operatorname{Ext}_{R}^{n}(J,)_{n \geq 0}$ also is a universal $\partial$-functor, again by $[2$, Proposition 2.2.1]. Therefore the natural equivalence $\theta: \operatorname{Ext}_{R}^{0}(J,) \rightarrow H^{0}(U[J] ; Q())$ can be extended (uniquely) to an equivalence of $\partial$-functors. In particular $\operatorname{Ext}_{R}^{n}(J, \quad)$ and $H^{n}(U[J] ; Q())$ are naturally equivalent functors, for each $n \geqq 0$.

4. Čech cohomology groups. A Hausdorff space $X$ will be called a Stone space if it is locally compact and totally disconnected. Equivalently, $X$ is an open subset of a Boolean space.

Theorem 4.1. Let $X$ be a Stone space, and let a be a sheaf of abelian groups over $X$. Then, for each $n \geqq 0, H^{n}(X ; Q)$ and $\check{H}^{n}(X: Q)$ are naturally isomorphic. Moreover, if $\mathcal{U}$ is any cover of $X$ consisting of compact open sets, the natural map: $H^{n}(\mathcal{U} ; Q) \rightarrow \check{H}^{n}(X ; Q)$ is an isomorphism, for each $n \geqq 0$.

Proof. Let $u$ be an arbitrary compact open cover of $X$. We first show that the sequence $H^{n}(\mathcal{U} ;)_{n \geq 0}$ forms a universal $\partial$-functor from $\mathcal{G}^{X}$ to $\mathrm{g}$. Again, we apply [2, Proposition 2.2.1]. Suppose

$$
0 \rightarrow a \rightarrow B \rightarrow \mathcal{C} \rightarrow 0
$$

is an exact sequence in $\mathcal{G}^{x}$. Then, for each $U \in \mathcal{U}$, the induced sequence

$$
0 \rightarrow \Gamma(U ; a) \rightarrow \Gamma(U ; B) \rightarrow \Gamma(U ; \mathcal{e}) \rightarrow 0
$$

is exact [4, Theorem 7.7]. It follows that the sequence of cochain complexes

$$
0 \rightarrow C(\mathcal{U} ; a) \rightarrow C(\mathcal{U} ; \mathfrak{B}) \rightarrow C(\mathcal{U} ; \mathcal{e}) \rightarrow 0
$$

is exact. The long exact sequence of cohomology now shows that $H^{n}(u ;)_{n \geq 0}$ forms an exact $\partial$-functor. It remains to be shown that $H^{p}(\mathcal{U} ;)$ is effaceable for $p>0$. If $a \in \mathcal{G}^{\mathbb{X}}$, let $\mathcal{F}$ be the first term of the canonical flabby resolution of $a$. Then $a$ is a subsheaf of $\mathcal{F}$, and $H^{p}(\mathcal{U} ; \mathcal{F})=0$, for $p>0$, [6, p. 106, Lemma 2]. Hence $H^{p}(\mathcal{U} ;)$ is effaceable.

Now suppose $v$ is a compact open cover which refines $\mathcal{u}$. The natural transformation $H^{0}(\mathcal{U} ;) \rightarrow H^{0}\left(\mathcal{V}_{;}\right)$is an equivalence, since both these functors are naturally equivalent to $\Gamma(X),,[6$, p. 106 , 
Lemma 3]. Then, since the natural transformations $H^{n}(\mathcal{U} ;)$ $\rightarrow H^{n}(U$; ) obviously form a morphism of $\partial$-functors, they must all be equivalences. The second statement in the theorem now follows, since $X$ has arbitrarily fine compact open covers. We now know that $\check{H}^{n}(X ;)_{n \geq 0}$ is a universal $\partial$-functor. Since $\check{H}^{0}(X ;)$ and $\Gamma(X, \quad)$ agree, the functors $\breve{H}^{n}(X ;)$ and $H^{n}(X ;)$ must be naturally equivalent, for each $n \geqq 0$. Q.E.D.

5. Examples. Recall that the sheaf dimension (or cohomological dimension) of a space $X$ is the sup of the integers $n$, such that there exists $a \in \mathcal{G}^{X}$, with $H^{n}(X ; a) \neq 0$. The following result, which depends on a theorem of Kaplansky [3], provides an easy method of obtaining Stone spaces with nontrivial cohomology:

Theorem 5.1. Let $X$ be a Stone space. Then sheaf $\operatorname{dim} X=0$ if and only if $X$ is a disjoint union of compact open sets.

Proof. Suppose $X$ is the disjoint union of a collection $u$ of compact open sets. Then $H^{p}(\mathcal{u} ; a)=0$, for each $p>0$ and each $a \in g^{x}$, by $[1$, II, 5.12]. Therefore sheaf $\operatorname{dim} X=0$, by Theorem 4.1. Conversely, suppose sheaf $\operatorname{dim} X=0$. Let $X^{*}$ be the one-point compactification of $X$. Let $R$ be the Boolean ring corresponding to $X^{*}$, and let $J$ be the ideal corresponding to $X$. Then, for each $R$-module $A$ and each $p>0, \operatorname{Ext}_{R}^{p}(J, A) \cong H^{p}(X ; Q(A))=0$, i.e., $J$ is projective. But then $J$ is a direct sum of principal ideals, by [3, Theorem 4]; hence $X$ is a disjoint union of compact open sets.

EXAmple 5.2. Let $X$ be the space of ordinals less than the first uncountable ordinal, with the order topology. Then $X$ is not a disjoint union of compact open sets. ${ }^{3}$ Hence sheaf $\operatorname{dim} X \neq 0$.

ExAmple 5.3.4 Let $S$ be a countable discrete space, and let $T$ be an uncountable discrete space. Let $S \cup\{p\}$ and $T \cup\{q\}$ be the onepoint compactifications of these spaces. Let $X=(S \cup\{p\}) \times(T \cup\{q\})$ $-\{(p, q)\}$. Then sheaf $\operatorname{dim} X \neq 0$, since $X$ is not normal, and hence not paracompact. ${ }^{5}$ Now let $u$ be the compact open cover of $X$ consisting of the sets $\{s\} \times(T \cup\{q\}), s \in S$, together with the sets $(S \cup\{p\}) \times\{t\}, t \in T$. Any three distinct sets in $u$ have empty intersection. It follows from $[1, I I, 5.12]$ that sheaf $\operatorname{dim} X=1$.

${ }^{3}$ It is well known that $X$ is not paracompact, and a disjoint union of compact open sets is obviously paracompact. In fact, it can be shown that a Stone space is paracompact if and only if it is a disjoint union of compact open sets.

- This example was suggested by Professor E. A. Michael.

- In fact, it can be shown that $H^{1}\left(X ; Z_{2}\right) \neq 0$. 
EXAMPLE 5.4. Let $\alpha$ be any infinite ordinal, and let $P$ be the product of $\boldsymbol{\aleph}_{\alpha}$ copies of the two-point discrete space $\{0,1\}$. Let $X$ be the space obtained by deleting a single point from $P$. Let $R$ be the Boolean ring corresponding to $P$, and let $J$ be the ideal corresponding to $X$. Then $J$ has infinite projective dimension [5]; hence sheaf $\operatorname{dim} X=\infty$.

\section{REFERENCES}

1. R. Godemont, Topologie algêbrique et theorie des faisceaux, Hermann, Paris, 1958.

2. A. Grothendieck, Sur quelque points d'algèbre homologique, Tôhoku Math. J. (2) 9 (1957), 119-221.

3. I. Kaplansky, Projective modules, Ann. of Math. 68 (1958), 372-377.

4. R. S. Pierce, Modules over commutative regular rings, Mem. Amer. Math. Soc. No. 70 (1967).

5. - The global dimension of Boolean rings, J. Algebra 7 (1967), 91-99.

6. R. G. Swan, The theory of sheaves, Univ. of Chicago Press, Chicago, Ill., 1964.

UNIVERSITY OF WISCONSIN 